JOURNAL OF PERIODONTAL RESEARCH doi:10.1111/j.1600-0765.2012.01479.x

\title{
Elevated levels of salivary lactoferrin, a marker for chronic periodontitis?
}

\begin{abstract}
Glimvall P, Wickström C, Jansson H. Elevated levels of salivary lactoferrin, a marker for chronic periodontitis? J Periodont Res 2012; 47: 655-660. (C) 2012 John Wiley \& Sons $A / S$
\end{abstract}

\begin{abstract}
Background and Objective: Whole saliva is a complex mixture of fluids essential for the well-being of the oral hard and soft tissues. Saliva contains numerous antimicrobial proteins that help protect the oral ecosystem from infectious agents. Chronic periodontitis is an infectious chronic inflammatory condition that affects the tooth-supporting structures and leads to their destruction. The aim of the present study was to investigate differences in concentrations of salivary lactoferrin in subjects with and without periodontal disease and correlate these values with clinical variables associated with periodontal disease.
\end{abstract}

Material and Methods: Stimulated whole saliva was collected from 17 subjects with chronic periodontitis and 17 periodontally healthy control subjects. Data relating to bleeding on probing, probing pocket depth and horizontal bone loss were registered. Concentrations of lactoferrin, lysozyme and IgA in stimulated whole saliva were quantified using ELISA.

Results: Subjects with chronic periodontits showed higher concentrations of lactoferrin in stimulated whole saliva compared with periodontally healthy control subjects $(p<0.05)$. Salivary concentrations of lactoferrin were positively correlated with bleeding on probing $(p<0.001)$ and the number of sites with probing pocket depth $\geq 6 \mathrm{~mm}(p<0.001)$.

Conclusion: Lactoferrin is raised in stimulated whole saliva in subjects with chronic periodontitis and is correlated with probing pocket depth $\geq 6 \mathrm{~mm}$.

\section{P. Glimvall ${ }^{1,2}$, C. Wickström ${ }^{2}$, H. Jansson ${ }^{1}$}

Departments of ${ }^{1}$ Periodontology and ${ }^{2}$ Oral Biology, Centre for Oral Health Sciences, Malmö University, Malmö, Sweden
Henrik Jansson, DDS, Odont Dr, Department of Periodontology, Faculty of Odontology, Malmö University, SE-205 06 Malmö, Sweden Tel: +46406658450 Fax: +46 406658405 e-mail: henrik.jansson@mah.se

Key words: periodontal risk factor; saliva; cytokine

Accepted for publication February 29, 2012
Chronic periodontitis is a chronic infectious inflammatory disease characterized by the destruction of toothsupporting structures (1). A limited number of specific bacteria are required, but not sufficient, for disease initiation (2). Disease onset and severity are determined by host factors such as heredity, diabetes and smoking (3). Diagnostics of periodontal disease is dependent to a great extent on clinical parameters such as probing pocket depth, clinical attachment loss, tooth mobility, furcation involvement, bleeding on probing, plaque index and radiographic quantification of marginal bone loss (4). These parameters mainly measure destruction from previous disease and tell us little about the patient's disease activity and risk for disease progression. Identification of individuals at risk of periodontal disease or disease progression has proved difficult both at a patient and at a tooth level. At a patient level, smoking and diabetes with poor glycaemic control appear to be the most significant predictors of progressive periodontitis (5).
Absence of bleeding on probing indicates periodontal stability, but its presence shows low specificity as an indicator of disease progression (6). The periodontal risk assessment model was developed in 2003 to help clinicians identify subjects at risk of further disease progression while in supportive periodontal treatment. The model incorporates six parameters associated with progression of periodontal disease (7). In 2008, this model was evaluated in subjects with severe periodontits enrolled in a supportive periodontal 
treatment programme. The authors concluded that the periodontal risk assessment model overestimates the risk for disease progression (8).

A diagnostic tool that would help clinicians to identify individuals with active disease and/or risk for disease progression would be of great value. With the knowledge of an individual's level of risk for disease or disease progression, clinicians could focus their treatment efforts on the individuals with the greatest need and avoid overtreatment of individuals with little risk of disease. One such possibility is the identification of one or more oral fluid biomarkers associated with periodontal disease and its progression. Oral fluid biomarkers related to periodontal disease can be associated with soft tissue inflammation, alveolar bone loss, bacterial products, antimicrobial proteins, etc. In an attempt to find biomarkers associated with periodontal disease and its progression, extensive studies have been done on crevicular fluid, glandular saliva and whole saliva (9-11). Of these fluids, stimulated whole saliva is the one most easily collected and contains proteins secreted from the salivary glands, as well as components from gingival crevicular fluid (12).

Lactoferrin is one of at least 45 different antimicrobial proteins identified in saliva (13). Lactoferrin is a metalloprotein that is produced in a number of tissues and is frequently found in human exocrine secretions such as tears, saliva, sweat, colostrum and milk. Polymorphonuclear leucocytes store large amounts of lactoferrin in their secretory granules (14). Faecal lactoferrin has been shown to be a sensitive and specific marker for inflammatory bowel disease and is useful in identifying patients with active disease (15-17). Faecal lactoferrin, together with faecal calprotectin, can be used to predict impending clinical relapse in inflammatory bowel disease (18). Lactoferrin has previously been examined in relation to periodontal disease, and higher concentrations of salivary lactoferrin have been shown in aggressive periodontitis $(19,20)$. In chronic periodontitis, higher concentrations of lactoferrin are found in gingival crevicular fluid and are correlated with probing pocket depth. The authors suggested that higher lactoferrin concentration in gingival crevicular fluid may be a more sensitive indicator of periodontal pathology compared with traditional clinical variables (21).

In analogy to faecal lactoferrin, we hypothesize that salivary lactoferrin can be used in the same way for periodontal disease, to identify subjects with periodontal disease and to differentiate subjects with active disease and/ or risk for disease progression. Therefore, the aim of the present study was to measure the concentration of lactoferrin in saliva from subjects with and without chronic periodontitis and test whether these concentrations correlate with clinical variables associated with periodontal disease.

\section{Material and methods}

\section{Subjects and inclusion criteria}

In order to be included in the study, test subjects were required to show general horizontal bone destruction of at least one-quarter of the root length as judged on intra-oral bitewing radiographs. Test subjects were further required to exhibit at least four teeth with pockets $\geq 5 \mathrm{~mm}$ and to be positive for bleeding on probing.

Individuals recruited to the control group were not allowed to exhibit any radiographic evidence of horizontal bone destruction as judged from intraoral bitewing radiographs. Furthermore, control subjects were not allowed to have any pockets $>4 \mathrm{~mm}$.

Sixty patients treated for moderately advanced chronic periodontits according to the definition by the American Academy of Periodontology 1999 (22) at the Department of Periodontology, Malmö University, Sweden were invited to take part in the study, which was conducted between October 2010 and December 2010. The subjects had all been referred for treatment of chronic periodontitis after initial screening at the Department of Oral Diagnostics, Malmö University, Sweden. Seventeen patients fulfilled the inclusion criteria.

For the control group, 50 patients treated for caries, endodontical and/or prosthodontic problems at the
Department of Prosthodontics, Malmö University, Sweden were also invited to take part in the study. Seventeen patients fulfilled the inclusion criteria. After sampling of whole stimulated saliva, patients were examined clinically and radiographically.

\section{Periodontal assessment}

All individuals underwent a periodontal examination by one examiner (P.G.), who had been calibrated for reproducibility before the study was conducted. A $\kappa$-value of $91 \%$ was calculated for probing pocket depth within $1 \mathrm{~mm}$. The following variables were analysed in the present study.

Number of teeth - A root remnant was considered as a missing tooth.

Probing pocket depth - All teeth were probed at four sites (mesiobuccal, midbuccal, distobuccal and midlingual). Only sites with probing $\geq 4 \mathrm{~mm}$ were registered. Probing was performed with a calibrated periodontal probe (PCP12; Hu-Friedy, Chicago, IL, USA) to the nearest millimetre (diameter of the probe tip was $0.5 \mathrm{~mm}$; $3 \mathrm{~mm}$ increments).

Bleeding on probing- Full-mouth bleeding on probing (23) was registered in conjunction with periodontal probing. If bleeding arose on probing, the examined site was considered inflamed.

Radiographic assessment to determine alveolar bone loss was undertaken as appropriate using bitewing and periapical radiographs.

\section{Sampling}

Two millilitres of stimulated whole saliva was collected while chewing on paraffin blocks in standardized conditions. The importance of standardized conditions and sample handling has been previously reported, and the use of stimulated saliva is recommended for ease of standardization $(24,25)$. Before sampling, subjects were required to chew for $1 \mathrm{~min}$, and saliva was discarded. Samples were frozen and stored at $-80^{\circ} \mathrm{C}$ until final analysis. 


\section{ELISA}

Salivary lactoferrin was analysed using indirect ELISA. In addition, human salivary IgA and lysozyme were analysed as a control. Samples were allowed to thaw at room temperature for $90 \mathrm{~min}$ and were then centrifuged at $18,000 \mathrm{~g}$ for $10 \mathrm{~min}$ in a centrifuge (Beckman Coulter, CA, USA) to remove particulates. After appropriate dilution in phosphate-buffered saline $\left(0.15 \mathrm{M} \mathrm{NaCl}+0.005 \mathrm{M} \mathrm{NaH}_{2} \mathrm{PO}_{4}\right.$, pH 6.5), samples were coated in duplicate onto multiwell assay plates (3912; Falcon, Franklin Lakes, NJ, USA) overnight at room temperature. Standards used were lactoferrin from human milk, lysozyme from neutrophils and IgA from colostrum (Sigma-Aldrich, St Louis, MO, USA). Plates were blocked for $1 \mathrm{~h}$ with phosphate-buffered saline containing $0.05 \%(\mathrm{v} / \mathrm{v})$ Tween 20 and $1 \%(\mathrm{w} / \mathrm{v})$ bovine serum albumin (blocking solution) and incubated $(1 \mathrm{~h})$. Reactivity was detected with an alkaline phosphatase-conjugated swine anti-rabbit antiserum, diluted 1:2000 in blocking solution using nitrophenyl phosphate $(2 \mathrm{mg} / \mathrm{mL}$ in $1 \mathrm{M}$ diethanolamine- $\mathrm{HCl}$ buffer, $\mathrm{pH}$ 9.8, containing $5 \mathrm{~mm}$ $\mathrm{MgCl}_{2}$ ) as a substrate. Reactivity was expressed as absorbances at $405 \mathrm{~nm}$ after $1 \mathrm{~h}$. Standards were serially diluted and used to generate standard curves. The curves showed a linear relationship between the serial diluted standards and the absorbance (data not shown). The amounts of the three proteins in the samples were determined by plotting the optical density against the separate standard curves and multiplied with dilution factors.

\section{Ethical requirements}

The study was performed in accordance with the Declaration of Helsinki. All study participants gave their signed, informed consent before inclusion in the project.

\section{Statistical analysis}

Statistical calculations were performed in InStat 3 (GraphPad Software Inc., La Jolla, CA, USA). Comparisons between the two groups were measured with Student's unpaired $t$-test (twotailed). Correlations were measured as Pearson product-moment correlation coefficient $(r)$. $p$-Values $<0.05$ were considered statistically significant.

\section{Results}

\section{Subjects}

In total, 60 subjects with chronic periodontitis $(\mathrm{CP}+)$ were examined and, of these, 17 subjects fulfilled the inclusion criteria and agreed to participate in the study. Fifty individuals without chronic periodontitis ( $\mathrm{CP}-$ ) were also examined and, of these, 17 subjects fulfilled the inclusion criteria and agreed to participate in the study. The study sample comprised 34 participants. The characterization of the study population by sex, age and periodontal status is presented in Table 1. Four of the 17 individuals in the $\mathrm{CP}-$ group had a bleeding on probing $>20 \%$, but without any bone loss. These individuals might be classified as patients with gingivitis. The $\mathrm{CP}+$ group contained eight smokers and five individuals with diabetes mellitus, while none of our control subjects were smokers or diabetics. Subjects with chronic periodontitis exhibited a higher bleeding on probing (45\%) compared with the control group $(20 \%)$. Sites with probing pocket depth $\geq 4 \mathrm{~mm}$ were prevalent in the test group, whilst only occasional sites with probing pocket depth $\leq 4 \mathrm{~mm}$ were found in the control group. Sites with probing pocket depth $\geq 6 \mathrm{~mm}$ were numerous and limited to the test group.

\section{Proteins}

In order to evaluate whether there are any associations between the lactoferrin concentration and chronic periodontitis, ELISA was used to measure the concentration of lactoferrin in our test and control groups. As a control, we also measured concentrations of $\mathrm{IgA}$ and lysozyme.

Concentrations for measured proteins can be found in Table 2. Significantly higher concentrations of lactoferrin were found in the test group $(2.71 \mu \mathrm{g} /$ $\mathrm{mL}$ ) compared with the control group $(1.12 \mu \mathrm{g} / \mathrm{mL} ; p<0.05)$. Our measurements of IgA and lysozyme showed no significant differences between the test group and control group.

Table 1. Clinical characteristics of subjects with $(\mathrm{CP}+)$ and without chronic periodontitis $(\mathrm{CP}-)$

\begin{tabular}{lrrc}
\hline & CP- & CP + & $p$-Value \\
\hline$n$ (female/male) & $17(12 / 5)$ & $17(6 / 11)$ & NS \\
Age (years) & $39 \pm 14$ & $58 \pm 11$ & $<0.001$ \\
Smoker & 0 & 8 & \\
Diabetes & 0 & 5 & \\
Number of teeth & $29 \pm 3$ & $23 \pm 4$ & $<0.001$ \\
Bleeding on probing (\%) & $20 \pm 15$ & $45 \pm 28$ & $<0.01$ \\
Number of sites with probing pocket depth 4-5 mm & $1 \pm 2$ & $26 \pm 17$ & $<0.001$ \\
Number of sites with probing pocket depth $\geq 6 \mathrm{~mm}$ & 0 & $14 \pm 13$ &
\end{tabular}

Values are expressed as means $\pm \mathrm{SD}$. The statistical method was Student's unpaired $t$-test. Abbreviation: NS, not significant.

Table 2. Salivary concentrations of lactoferrin, lysozyme and $\operatorname{IgA}$ in subjects with $(\mathrm{CP}+)$ and without chronic periodontitis $(\mathrm{CP}-)$

\begin{tabular}{lccl}
\hline & $\mathrm{CP}-(n=17)$ & $\mathrm{CP}+(n=17)$ & $p$-Value \\
\hline Lactoferrin $(\mu \mathrm{g} / \mathrm{mL})$ & $1.12 \pm 1.98$ & $2.71 \pm 2.36$ & $p<0.05$ \\
Lysozyme $(\mu \mathrm{g} / \mathrm{mL})$ & $0.42 \pm 0.32$ & $0.37 \pm 0.21$ & $\mathrm{NS}$ \\
$\operatorname{IgA}(\mu \mathrm{g} / \mathrm{mL})$ & $518.07 \pm 665.65$ & $283.90 \pm 217.68$ & $\mathrm{NS}$ \\
\hline
\end{tabular}

Values are expressed as means \pm SD. The statistical method used was Student's unpaired $t$-test. Abbreviation: NS, not significant. 
Table 3. Pearson product-moment correlation coefficient $(r)$ for salivary protein concentration and clinical variables associated with periodontal disease in individuals with $(\mathrm{CP}+)$ and without chronic periodontitis $(\mathrm{CP}-)$

\begin{tabular}{|c|c|c|c|c|c|c|}
\hline \multirow[b]{2}{*}{ Protein } & \multicolumn{2}{|c|}{ Bleeding on probing } & \multicolumn{2}{|c|}{ Probing pocket depth $\geq 4-5 \mathrm{~mm}$} & \multicolumn{2}{|c|}{ Probing pocket depth $\geq 6 \mathrm{~mm}$} \\
\hline & $\mathrm{CP}-(n=17)$ & $\mathrm{CP}+(n=17)$ & $\mathrm{CP}-(n=17)$ & $\mathrm{CP}+(n=17)$ & $\mathrm{CP}-(n=17)$ & $\mathrm{CP}+(n=17)$ \\
\hline Lactoferrin & $0.49^{*}$ & $0.77 * * *$ & 0.25 & 0.45 & - & $0.75^{* * *}$ \\
\hline Lysozyme & 0.04 & 0.34 & -0.20 & 0.39 & - & -0.15 \\
\hline IgA & 0.02 & 0.29 & -0.16 & 0.17 & - & 0.27 \\
\hline
\end{tabular}

The statistical method used was Student's unpaired $t$-test.

${ }^{*} p<0.05,{ }^{* *} p<0.01$ and ${ }^{* * *} p<0.001$.

Pearson product-moment correlation coefficient for protein concentration and clinical variables associated with periodontal disease are presented in Table 3 and Fig. 1. Lactoferrin was positively correlated with bleeding on probing in the test group $(p<0.001)$ and in the control group $(p<0.05)$. In the test group, concentrations of salivary lactoferrin were positively correlated with the number of sites with probing pocket depth $\geq 6 \mathrm{~mm}$ $(p<0.001)$. Neither IgA nor lysozyme showed any significant correlation with the tested variables.

\section{Discussion}

The key finding of the present study was that individuals with chronic peri-
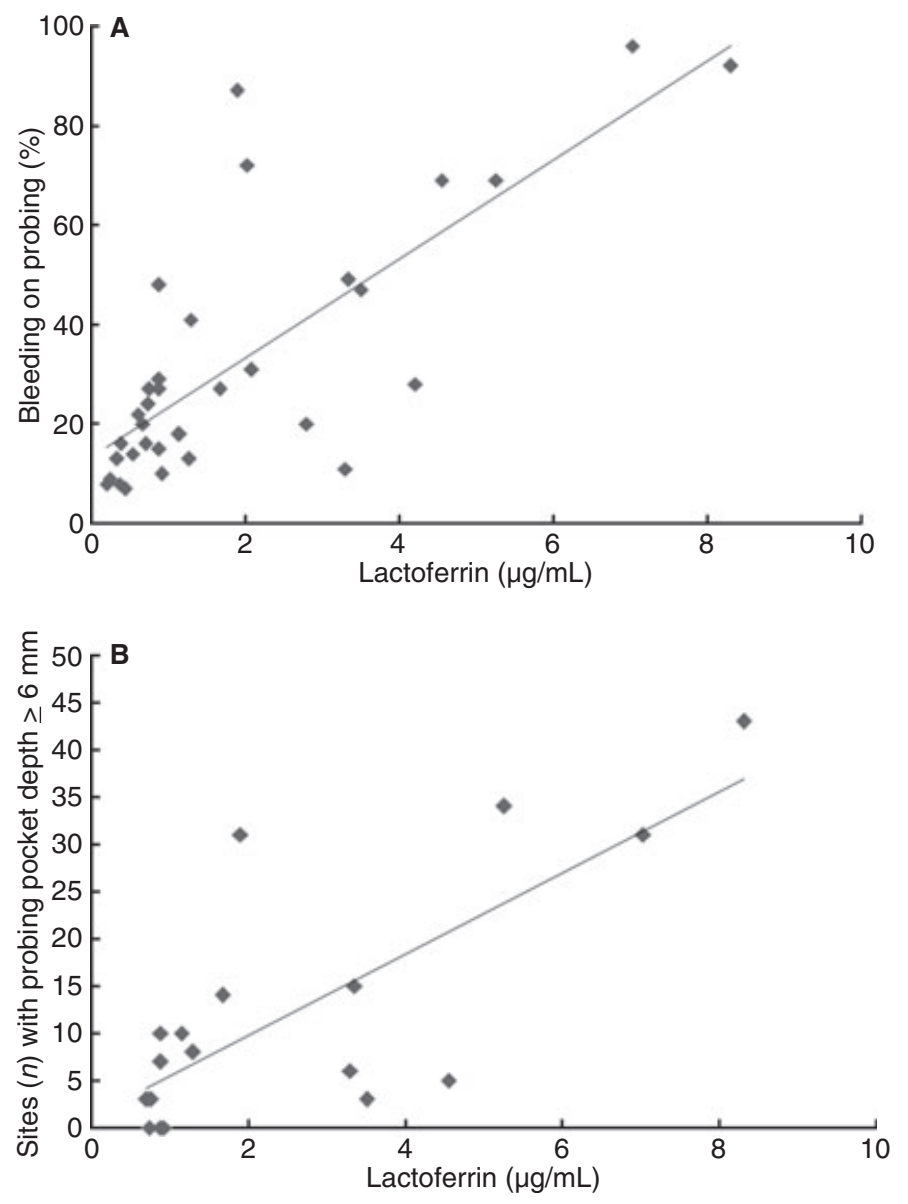

Fig. 1. (A) Correlation between salivary concentration of lactoferrin (in micrgrams per millilitre) and bleeding on probing (as a percentage). $r=0.77, p<0.001$. (B) Correlation between salivary concentration of lactoferrin (in micrgrams per millilitre) and sites $(n)$ with probing pocket depth $\geq 6 \mathrm{~mm} . r=0.75, p<0.001$.

odontitis had higher concentrations of salivary lactoferrin in stimulated whole saliva. Elevated concentrations of salivary lactoferrin have previously been reported in subjects with aggressive periodontitis $(19,20)$, but to our knowledge, this is the first study showing this in patients with chronic periodontitis.

The increased levels of lactoferrin found in this paper can be explained by increased secretion from the salivary glands and/or increased leakage from the crevicular fluid. Sites with periodontal disease have been shown to have larger volumes of crevicular fluid compared with healthy sites and also a higher concentration of lactoferrin $(21,26)$. The crevicular fluid concentration of lactoferrin has been shown to decrease towards normal concentrations after treatment, which suggests that lactoferrin might be a suitable marker for monitoring treatment effects in periodontal disease $(26,27)$. In the present study, we cannot exclude a contribution of crevicular lactoferrin to the higher concentrations found in $\mathrm{CP}+$ subjects; however, as we used stimulated whole saliva, comparing the relative amounts originating from the major salivary glands with the crevicular fluid contribution, it is reasonable to assume that the major part of the increase in lactoferrin concentration originates from the salivary glands. This, however, needs to be further studied before a definite conclusion can be drawn. Lactoferrin originating from the salivary glands has been shown to be present at higher concentrations in subjects with aggressive periodontitis when both whole and parotid saliva were analysed (20).

In the $\mathrm{CP}+$ group in this study, a few subjects were smokers and some 
suffered from diabetes, facts that might impair the validity of the results. However, studies concerning smoking and concentrations of lactoferrin in saliva have shown that lactoferrin levels tend to decrease in smokers (28-30). The expected influence of including smokers in this study would be that we might underestimate the increase of lactoferrin concentration within the $\mathrm{CP}+$ group. For patients with diabetes, both higher concentrations of salivary lactoferrin have been found (31), as well as no differences in concentration (32) when compared with healthy control subjects. In the present study, we found significantly increased levels of lactoferrin, despite the presence of smokers and diabetic patients in the $\mathrm{CP}+$ group. In addition, when the smokers and diabetic patients were excluded from the results, the significance still remained.

In this study, salivary concentrations of lactoferrin were positively correlated with bleeding on probing in both groups. This correlation was most prominent in the group with chronic periodontitis. In the $\mathrm{CP}+$ group, salivary lactoferrin was also correlated with the number of sites with probing pocket depth $\geq 6 \mathrm{~mm}$. Correlations between salivary lactoferrin and bleeding on probing in adolescents have been found previously (33). Residual sites with probing pocket depth $\geq 6 \mathrm{~mm}$ after initial periodontal therapy have been associated with risk for disease progression and tooth loss in subjects enrolled in a supportive periodontal therapy programme $(34,35)$. Although it requires further study, lactoferrin concentrations in saliva could also be a candidate marker for disease progression.

In inflammatory bowel disease, which is a chronic relapsing inflammatory disorder, thought to result from an inappropriate inflammatory response to commensal microbes in a susceptible host (36), faecal lactoferrin is used as a diagnostic marker. Inflammatory bowel disease and periodontal disease share several factors in their aetiology and pathogenesis $(36,37)$, and studies have shown that faecal lactoferrin can be used to identify subjects with inflammatory bowel disease and also to distinguish between active and inactive disease $(15,16)$. It is tempting to speculate that salivary lactoferrin could be used to assess disease activity in chronic periodontitis.

Two control proteins, lysozyme and IgA, were analysed in conjunction with lactoferrin. No differences in the concentration of these two proteins were seen between the two groups. These findings show that the differences in lactoferrin levels presented here are not due to a generally altered protein concentration. Lysozyme has been studied previously, and no correlation between lysozyme concentration and bleeding on probing was found in adolescents (33). Concerning salivary $\operatorname{IgA}$ and periodontal disease, both higher $(38,39)$ and lower $(40,41)$ concentrations of salivary IgA have been shown in subjects with periodontal disease.

In conclusion, higher levels of salivary lactoferrin were detected in subjects with chronic periodontits and correlated with probing pocket depth $\geq 6 \mathrm{~mm}$. Further studies with a prospective and longitudinal design are required to determine whether salivary lactoferrin can be used as a diagnostic marker for periodontal disease and/or as a prognostic marker for disease progression. Another valuable aspect is to establish whether salivary lactoferrin can be confirmed as a biological marker of active and inactive periodontal disease.

\section{Acknowledgements}

None.

\section{References}

1. Tonetti MS, Claffey N; European Workshop in Periodontology group C. Advances in the progression of periodontitis and proposal of definitions of a periodontitis case and disease progression for use in risk factor research. Group C consensus report of the 5th European Workshop in Periodontology. $J$ Clin Periodontol 2005;32:210-213.

2. Graves DT, Fine D, Teng YT, Van Dyke TE, Hajishengallis G. The use of rodent models to investigate host-bacteria interactions related to periodontal diseases. J Clin Periodontol 2008;35:89-105.

3. Page RC, Offenbacher S, Schroeder HE, Seymour GJ, Kornman KS. Advances in the pathogenesis of periodontitis: summary of developments, clinical implications and future directions. Periodontol 2000 1997;14:216-248.

4. Armitage GC. The complete periodontal examination. Periodontol 2000 2004;34: 22-33.

5. Heitz-Mayfield LJ. Disease progression: identification of high-risk groups and individuals for periodontitis. J Clin Periodontol 2005;32:196-209.

6. Lang NP, Joss A, Orsanic T, Gusberti FA, Siegrist BE. Bleeding on probing. A predictor for the progression of periodontal disease?. J Clin Periodontol 1986;13:590596.

7. Lang NP, Tonetti MS. Periodontal risk assessment (PRA) for patients in supportive periodontal therapy (SPT). Oral Health Prev Dent 2003;1:7-16.

8. Jansson H, Norderyd O. Evaluation of a periodontal risk assessment model in subjects with severe periodontitis. A 5-year retrospective study. Swed Dent J 2008;32:1-7.

9. Fine DH, Mandel ID. Indicators of periodontal disease activity: an evaluation J Clin Periodontol 1986;13:533-546.

10. Giannobile WV, Beikler T, Kinney JS, Ramseier CA, Morelli T, Wong DT. Saliva as a diagnostic tool for periodontal disease: current state and future directions. Periodontol 2000 2009;50:52-64.

11. Kaufman E, Lamster IB. Analysis of saliva for periodontal diagnosis-a review. $J$ Clin Periodontol 2000;27:453-465.

12. Edgar WM. Saliva: its secretion, composition and functions. Br Dent $J$ 1992;172: 305-312.

13. Gorr SU. Antimicrobial peptides of the oral cavity. Periodontol 2000 2009;51:152180.

14. Azevedo LF, Pecharki GD, Brancher JA et al. Analysis of the association between lactotransferrin (LTF) gene polymorphism and dental caries. J Appl Oral Sci 2010;18:166-170.

15. Walker TR, Land ML, Kartashov A et al. Fecal lactoferrin is a sensitive and specific marker of disease activity in children and young adults with inflammatory bowel disease. $J$ Pediatr Gastroenterol Nutr 2007;44:414-422.

16. Sidhu R, Wilson P, Wright A et al. Faecal lactoferrin-a novel test to differentiate between the irritable and inflamed bowel? Aliment Pharmacol Ther 2010;31:13651370.

17. Jones J, Loftus EV Jr, Panaccione R et al. Relationships between disease activity and serum and fecal biomarkers in patients with Crohn's disease. Clin Gastroenterol Hepatol 2008;6:1218-1224.

18. Gisbert JP, Bermejo F, Perez-Calle JL et al. Fecal calprotectin and lactoferrin for the prediction of inflammatory bowel 
disease relapse. Inflamm Bowel Dis 2009;15:1190-1198.

19. Groenink J, Walgreen-Weterings E, Nazmi K et al. Salivary lactoferrin and low-Mr mucin MG2 in Actinobacillus actinomycetemcomitans-associated periodontitis. J Clin Periodontol 1999;26:269275.

20. Fine DH, Furgang D, Beydouin F. Lactoferrin iron levels are reduced in saliva of patients with localized aggressive periodontitis. J Periodontol 2002;73:624630.

21. Tsai CC, Kao CC, Chen CC. Gingival crevicular fluid lactoferrin levels in adult periodontitis patients. Aust Dent J 1998; 43:40-44.

22. Flemmig TF. Periodontitis. Ann Periodontol 1999;4:32-38.

23. Mühleman $\mathrm{H}$, Son $\mathrm{S}$. Gingival sulcus index. A leading symptom in initial gingivitis. Helv Odontol Acta 1971;15:107113.

24. Al-Tarawneh SK, Border MB, Dibble CF, Bencharit S. Defining salivary biomarkers using mass spectrometry-based proteomics: a systematic review. OMICS 2011;15:353-361.

25. Kawas SA, Rahim ZH, Ferguson DB. Potential uses of human salivary protein and peptide analysis in the diagnosis of disease. Arch Oral Biol 2012;57:1-9.

26. Suomalainen K, Saxen L, Vilja P, Tenovuo J. Peroxidases, lactoferrin and lysozyme in peripheral blood neutrophils, gingival crevicular fluid and whole saliva of patients with localized juvenile periodontitis. Oral Dis 1996;2:129-134.
27. Jentsch H, Sievert Y, Gocke R. Lactoferrin and other markers from gingival crevicular fluid and saliva before and after periodontal treatment. $J$ Clin Periodontol 2004;3:511-514.

28. Nishida N, Yamamoto $\mathrm{Y}$, Tanaka M et al. Association between involuntary smoking and salivary markers related to periodontitis: a 2-year longitudinal study. J Periodontol 2008;79:2233-2240.

29. Kibayashi M, Tanaka M, Nishida N et al. Longitudinal study of the association between smoking as a periodontitis risk and salivary biomarkers related to periodontitis. J Periodontol 2007;78:859-867.

30. Olson BL, McDonald JL, Gleason MJ et al. Comparisons of various salivary parameters in smokers before and after the use of a nicotine-containing chewing gum. J Dent Res 1985;64:826-830.

31. Dodds MW, Yeh CK, Johnson DA Salivary alterations in type 2 (non-insulindependent) diabetes mellitus and hypertension. Community Dent Oral Epidemiol 2000;28:373-381.

32. Tabak L, Mandel ID, Karlan D, Baurmash H. Alterations in lactoferrin in salivary gland disease. J Dent Res 1978; 57:43-47.

33. Jalil RA, Ashley FP, Wilson RF, Wagaiyu EG. Concentrations of thiocyanate, hypothiocyanite, 'free' and 'total' lysozyme, lactoferrin and secretory $\operatorname{IgA}$ in resting and stimulated whole saliva of children aged 12-14 years and the relationship with plaque accumulation and gingivitis. J Periodontal Res 1993;28:130-136.
34. Joss A, Adler R, Lang NP. Bleeding on probing. A parameter for monitoring periodontal conditions in clinical practice. J Clin Periodontol 1994;21:402-408.

35. Matuliene G, Pjetursson BE, Salvi GE et al. Influence of residual pockets on progression of periodontitis and tooth loss: results after 11 years of maintenance. $J$ Clin Periodontol 2008;35:685-695.

36. Khor B, Gardet A, Xavier RJ. Genetics and pathogenesis of inflammatory bowel disease. Nature 2011;474:307-317.

37. Indriolo A, Greco S, Ravelli P, Fagiuoli S. What can we learn about biofilm/host interactions from the study of inflammatory bowel disease. $J$ Clin Periodontol 2011;38:36-43.

38. Bachrach G, Muster Z, Raz I et al. Assessing the levels of immunoglobulins in the saliva of diabetic individuals with periodontitis using checkerboard immunodetection. Oral Dis 2008;14:51-59.

39. Sandholm L, Gronblad E. Salivary immunoglobulins in patients with juvenile periodontitis and their healthy siblings. J Periodontol 1984;55:9-12.

40. Hagewald S, Bernimoulin JP, Kottgen E, Kage A. Salivary IgA subclasses and bacteria-reactive $\operatorname{IgA}$ in patients with aggressive periodontitis. $J$ Periodontal Res 2002;37:333-339.

41. Hagewald S, Bernimoulin JP, Kottgen E, Kage A. Total IgA and Porphyromonas gingivalis-reactive $\operatorname{IgA}$ in the saliva of patients with generalised early-onset periodontitis. Eur J Oral Sci 2000;108:147153. 